\title{
Non-coding RNAs make E. coli unpalatable
}

Although food intake is known to influence gene expression and morphology in animals, in general the mechanisms underlying these effects are unknown. Writing in Nature Communications, Liu et al. now show that endogenous non-coding RNAs (ncRNAs) from Escherichia coli can modulate gene expression in Caenorhabditis elegans to protect the bacterium from foraging by the worm.

It has long been appreciated that the bacterial food on which C. elegans is fed can be used to influence gene expression in the worm through the inclusion of recombinant $E$. coli that expresses double-stranded RNAs (dsRNAs), which act as an environmental source for RNAi. Given the effects of these 'artificial' RNAs, the authors speculated that endogenous bacterial



RNAs, and in particular small, regulatory ncRNAs, might also influence the nematode.

They began by investigating OxyS, a small ncRNA that is produced by E. coli under oxidative stress conditions and regulates bacterial gene expression. Using E. coli strains that constitutively expressed OxyS, the authors showed that although the OxyS-expressing bacteria were found and eaten by the nematode when they were present as the sole food source, the nematode did not remain on these bacteria when given the choice between these and wild-type E. coli. Furthermore, worms fed OxyS-expressing bacteria were impaired for both chemo-attraction to sodium chloride and chemoavoidance of copper acetate, indicating that OxyS interferes with chemosensing in the worm, thus impeding food searching.

The authors identified 17 nucleotides of complementarity to OxyS in che-2 (a C. elegans gene involved in chemosensing and food searching), suggesting that the effects of OxyS are mediated by RNA silencing. In agreement with this hypothesis, worms fed OxyS-expressing E. coli had less che-2 mRNA than worms fed wild-type E. coli, but there was no difference in the expression of GFP from the che-2 promoter in either group. The authors therefore concluded that OxyS impairs chemosensing and food searching in C. elegans by reducing nematode che-2 mRNA levels, probably through mRNA decay rather than inhibition of transcription.

In addition to the effects of Oxys, feeding C. elegans with E. coli expressing another bacterial ncRNA, DsrA, caused a decrease in the levels of nematode F42G9.6 mRNA (which encodes a diacylglycerol lipase orthologue) and led to a reduction in the longevity of the worm. Finally, the interspecies effects of both ncRNAs required $r d e-4$, which encodes a C. elegans-specific dsRNA-binding protein that is involved in RNAi initiation.

Although E. coli is not thought to be a major food source for $C$. elegans in the wild, it is possible that these sequences evolved to protect the bacterium against opportunistic foraging or against feeding during suboptimal conditions. Indeed, the authors isolated both species from three rotten plant samples, indicating that the two do co-exist in nature. It will be interesting to see whether other bacterial species that are major food sources for C. elegans have similar ncRNAs with similar interspecies regulatory functions.

Lucie Wootton

ORIGINAL RESEARCH PAPER Liu, H. et al. Escherichia coli noncoding RNAs can affect gene expression and physiology of Caenorhabditis elegans. Nature Commun. 3, 1073 (2012) 